\title{
The Effects of a Pain Management-Focused Mobile Health Behavior Intervention on Older Adults' Self-efficacy, Satisfaction with Functioning, and Quality of Life: a Randomized Pilot Trial
}

\author{
J. Fanning ${ }^{1}$ (D) A. K. Brooks ${ }^{2}$ - K. L. Hsieh ${ }^{1}$ 'K. Kershner ${ }^{1}$ J. Furlipa ${ }^{1}$ - B. J. Nicklas ${ }^{3}$ - W. J. Rejeski ${ }^{1}$
}

Accepted: 14 May 2021 / Published online: 20 May 2021

(c) International Society of Behavioral Medicine 2021

\begin{abstract}
Background This report investigates the impact of a remote physical activity intervention on self-efficacy, satisfaction with functioning, and health-related quality of life (HRQOL) as assessed by the SF-36 in obese older adults with chronic pain. The intervention was group-mediated in nature and based in social cognitive theory and mindfulness-based relapse prevention. Methods Participants $(N=28 ; 70.21 \pm 5.22$ years) were randomly assigned to receive either the active intervention, which focused on reducing caloric intake and increasing steps across the day or to a waitlist control condition.

Results Over 12 weeks, intervention participants reported a moderate, positive improvement in self-efficacy for walking relative to control. They also reported large magnitude improvements in satisfaction for physical functioning as well as improvements on pain and the physical functioning subscales of the SF-36.

Conclusions These findings expand on previous research showing similar effects in response to structured exercise, this time via a protocol that is likely to be scalable and sustainable for many older adults. Additional work on larger and more diverse samples is warranted.
\end{abstract}

Keywords Aging $\cdot$ MHealth $\cdot$ Weight loss $\cdot$ Physical activity $\cdot$ Telehealth

\section{Introduction}

The number of obese older adults in the USA is increasing rapidly, mirroring both the growing proportion of older adults in the USA and the increasing prevalence of obesity in this population [1]. Obesity is costly in that it exaggerates increases in healthcare utilization with aging, negatively impacts health-related quality of life (HRQOL [2]), and reduces independence. Moreover, it is especially troubling for those with chronic pain, as it exacerbates pain symptoms [3]. Behavioral weight loss can help to combat pain and enhance physical function, HRQOL, and longevity [4].

J. Fanning

Fanninjt@wfu.edu

1 Department of Health and Exercise Sciences, Wake Forest University, Winston-Salem, NC, USA

2 Department of Anesthesiology, Wake Forest School of Medicine, Winston-Salem, NC, USA

3 Department of Internal Medicine, Wake Forest School of Medicine, Winston-Salem, NC, USA
However, losing weight and increasing physical activity, even in the absence of pain, are a challenging behavioral goal that acts against humans' evolutionary need to retain energy [5]. Behavior change requires that individuals build a robust sense of self-efficacy, develop social ties, and identify intrinsically pleasing methods of moving and eating healthfully [6-8]. The 12-week pilot Mobile Health Intervention to Reduce Pain and Improve Health (MORPH) trial tested a remote social cognitive theory-driven physical activity program for older adults with chronic pain. Those who received the program demonstrated less pain intensity and better weight loss and physical function compared with a control condition [9]. What follows is a secondary analysis of MORPH on selfefficacy, satisfaction with physical functioning, and HRQOL in older adults with chronic multisite pain.

Diet and physical activity are unique and effortful behaviors that are susceptible to both stable (e.g., poor self-efficacy) and dynamic (e.g., pain) barriers $[10,11]$. Self-efficacy is a key cause and consequence of behavioral adoption and maintenance, reflecting an individual's perceived ability to successfully bring about a specific course of action [7]. The model underlying the 12-week MORPH pilot randomized controlled 
trial centered on promotion of self-efficacy via self-monitoring, immediate and specific goals, and progressive goal attainment (see [12] for more information). As sustained sitting can contribute to pain, this protocol involved the promotion of stepping across the day driven by a custom smartphone app and an activity monitor, and participants aimed to find methods of moving that were intrinsically pleasing and attainable. This was paired with a well-tested group-mediated dietary weight loss intervention [13] that was delivered primarily via teleconference software. During each weekly session, participants engaged in a brief mindfulness strategy adapted from mindfulness-based relapse prevention [14] designed to support behavior change in the face of pain and other challenging moments. We have previously reported that this protocol was successful in promoting weight loss, reducing sitting time, improving function, and reducing pain intensity over 12 weeks relative to a control condition [9].

Supporting HRQOL is an important outcome in geriatric medicine that is closely tied to self-efficacy, physical functioning, and one's perception of their functional capabilities $[2,4,15,16]$. It is influenced by the promotion of physical activity and purposeful weight loss $[4,17]$. Several research teams have demonstrated that the impact of physical activity on HRQOL is at least partially indirect via self-efficacy [4, 16]. Research to date has investigated the impact of promoting structured, purposeful aerobic and resistance training on HRQOL, self-efficacy, and satisfaction with functioning [18]. Determining whether remote non-exercise activity and weight loss interventions yield beneficial effects on these key social cognitive outcomes is an important early step in the science of behavior change [19]. In this secondary analysis of MORPH, we hypothesized that those who received the intervention would report greater self-efficacy and satisfaction after 12 weeks compared to those in the control. We have previously reported beneficial effects of weight loss plus structured exercise on physical subcomponents of HRQOL [18]; thus, we hypothesized that MORPH participants would report improved scores in these domains (i.e., physical functioning, pain, general health, role limitations due to physical health problems, general perceptions of health).

\section{Methods}

\section{Participants}

The methods and CONSORT diagram for MORPH have been provided in detail elsewhere [9, 12]. Participants were recruited between March 2018 and October 2019. Eligible individuals were 55-85 years of age, had a body mass index (BMI) of $30-45 \mathrm{~kg} / \mathrm{m}^{2}$, did not engage in regular resistance training and/or more than $20 \mathrm{~min}$ of aerobic exercise on 2 or more days per week in the previous 6 months, had not lost or gained more than $5 \%$ of their body weight in previous 6 months, had no contra-indication to exercise, owned a smartphone, and had pain in at least two of the following sites on most days in the previous 3 months: back, neck, shoulders, hips, or knees.

\section{Intervention Procedures}

Interested individuals first completed a telephone screening, and those who were eligible to participate attended a baseline testing session wherein eligibility was verified, and baseline questionnaires were collected via interview with research staff (see Fig. 1 for a flow diagram). Following this session, participants were randomized using a web-based randomization scheme developed by the study biostatistician in a 1:1 ratio to either the active intervention or a waitlist control. Those in the intervention condition attended a second baseline appointment wherein they were introduced to the program and received a selfefficacy-driven technology orientation. Here, participants

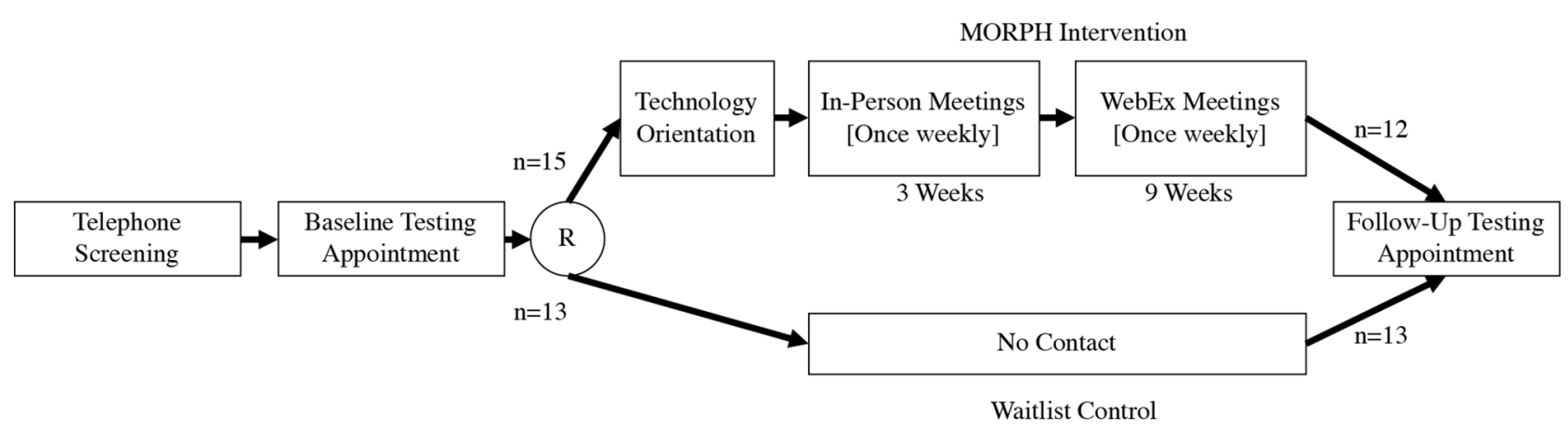

Fig. 1 Study flow diagram. Reasons for attrition include $(n=1)$ expected MORPH to include pharmaceutical intervention, $(n=1)$ death of a family member, and $(n=1)$ caregiving responsibilities.
Note that control participants received the full MORPH program following completion of all testing procedures 
received all technological tools including the Fitbit Alta activity monitor and Fitbit app, BodyTrace 4G cellular scale, and the MORPH Companion App. Participants were introduced to the Fitbit app, which transmitted data to the study app, and the Companion App, which was meant to help participants self-monitor movement throughout the day, engage with study staff and group members, and receive immediate feedback on goal achievement; see [12] for details. Participants were asked to navigate each app, attempting to interact with all app features while narrating their inner dialogue aloud. The study staff member remained quiet unless a participant was stuck, at which point they would guide them toward the next feature. This process is meant to instill confidence in exploring the tools while providing practice in key smartphone interactions (touch, swipe, hold). The session concluded with a debrief of each core app feature.

The focus of the MORPH program was managing pain through weight loss, the development of mindful awareness [14], and frequent bouts of movement. To facilitate this, participants met in small groups of their peers, led by a registered dietician and a trained behavioral coach, once weekly. These were held in-person for the first 3 weeks to develop social bonds, and then via teleconference software for the remaining nine weeks. Each session was designed to last approximately $1 \mathrm{~h}$, including goal review and revision, education on the importance of weight and movement for pain management and quality of life, and practice in basic mindfulness skills [14]. These sessions also focused on group discussion of successes and group troubleshooting of barriers as they arose. Weekly calorie goals were tailored by the dietician using data collected from the cellular weight scale and participants aimed to achieve an approximate $400 \mathrm{kcal} / \mathrm{day}$ deficit from daily weight requirements (minimum caloric intake goal of $1100 \mathrm{kcal} / \mathrm{day}$ for women and $1200 \mathrm{kcal} / \mathrm{day}$ for men). Movement goals centered on the accumulation of Fitbit-recorded steps throughout the day to break sustained sitting time. Participant Fitbit data were streamed into the Companion app (see Supplemental Fig. 1) and visualized on a timeline bar as blue periods of sitting and green periods of movement. Participants aimed to minimize the presence of sustained blue sitting. They also aimed to work toward a daily stepping goal that was tailored by the behavioral coach each week with a maximal stepping goal of 10,000 daily "periodic" steps that were designed to encourage movement throughout the day. In brief, participants were able to earn up to $45 \%$ of their daily stepping goal (e.g., 4,500 of 10,000 steps) before noon, again between noon and 5:30 pm, and once more after 5:30 pm. This required a minimum of $10 \%$ of the daily step goal during any period in order to achieve the overall daily step goal. Participants attended one final testing appointment on completion of the 12-week study. All assessments were collected by blinded research stuff.

\section{Measures}

Self-efficacy for walking [20] was measured using an 8-item scale wherein participants noted their confidence in their ability to walk over incrementally longer durations without stopping. Responses were provided on an 11-point Likert scale ( $0=$ "not at all confident," $10=$ "highly confident"). Internal consistency for this scale in the present study was excellent $(\mid \alpha=0.956-0.963)$. We measured satisfaction with various components of physical functioning ([21]; e.g., "how satisfied are you with your overall level of physical fitness") on a 7-item scale ( 1 = "very dissatisfied," 7 = "very satisfied"). These items are summed to produce a final scale score. Internal consistency for this scale was good-toexcellent $(\mid \alpha=0.878-0.949)$. Finally, health-related quality of life was measured using the 36-Item Short Form Survey (SF-36). This widely used measure taps eight domains of health-related quality of life, including physical functioning, bodily pain, role limitations due to physical health problems, role limitations due to personal or emotional problems, emotional well-being, social functioning, energy and fatigue, and perceptions of general health. Each subscale produces a score ranging from 0 to 100 such that higher scores represent a more favorable health state [22]. The SF-36 has been demonstrated to be a valid measure of HRQOL across many clinical populations including older adults and those with chronic pain [23].

\section{Analyses}

Participant characteristics were summarized using mean (SD) for continuous measures and $\mathrm{n}(\%)$ for count measures. The effect of MORPH on self-efficacy for walking, satisfaction with physical functioning, and each SF-36 subscale was assessed via mixed analysis of variance (ANOVA) models including group, time (baseline, week 12), and their interaction as fixed factors, controlling for age and sex due to their association with levels of physical activity and sedentary behavior [24]. In light of our small sample size, we focus our interpretation on $\mid \eta^{2}$ effect sizes following Cohen's guidelines ([25], pp. 285-288) such that $\mid \eta^{2}=0.01$ suggests a small effect, $\mid \eta^{2}=0.06$ suggests a medium effect, and $\mid \eta^{2}=0.14$ suggests a large effect. For interactions of a medium or large size, we provide adjusted scores in-text to aid in interpretation. $P$ values are provided in tables for reference. Each model was checked for outliers and normality via $\mathrm{Z}$ scores and transformed as necessary. Of note, the emotional well-being subscale did demonstrate a kurtotic $(\mathrm{Z}=3.12)$ and negatively skewed distribution $(\mathrm{Z}=-2.59)$ in the intervention condition at baseline. A reflected log transformation was successful in bringing these $\mathrm{Z}$ scores within range but did not meaningfully affect interpretation. Satisfaction with function scores was also positively skewed 
( $Z s \geq 3.05)$, so scores were log-transformed. Three of 15 participants had missing data in the intervention condition, so expectation maximization [26] was used to impute values, and the results are presented in Supplemental Table 1 and discussed in-text where they differ from the main analyses.

\section{Results}

Participant characteristics are displayed in Table 1. In total, 223 participants were screened, and 28 participants were eligible and agreed to participate (15 intervention; 70.21 \pm 5.22 years). Participants had an average baseline BMI of $36.96 \pm 4.54 \mathrm{~kg} / \mathrm{m}^{2}$. Twenty-two $(78.6 \%)$ were female, 23 $(82.1 \%)$ were white, $24(85.7 \%)$ had at least a college degree, and $100 \%$ of control participants and 12 of 15 intervention participants (80.0\%) completed follow-up testing. For a description of participants' pain symptoms at study entry and following intervention, see [9].

Regarding self-efficacy for walking, there was a moderate effect for the interaction between time and condition; $\mathrm{F}(1$, $21)=2.165, \eta^{2}=0.093$ (Table 2). Examination of marginal means indicates a decrease in self-efficacy of $0.9 \%$ in control and an increase in self-efficacy of $14.2 \%$ in the intervention condition. We observed a large time $\times$ condition effect for satisfaction with function, $F(1,21)=4.599, \eta^{2}=0.180$. This was driven by a $4.7 \%$ increase in satisfaction among those in the intervention condition, and an increase of $0.8 \%$ in the control condition. For the physical functioning subscale of the SF-36, the group by time interaction yielded a large effect; $F(1,21)=3.787, \eta^{2}=0.153$. Examination of marginal means revealed an increase in 13.38 points in the intervention condition and a decrease of 4.28 points in the control condition. Additionally, there was a large group by time interaction for the SF-36 pain subscale; $F(1,21)=4.265, \eta^{2}$
$=0.169$. Examination of marginal means indicates this was driven by an increase of 15.13 points in the intervention condition compared with an increase of 0.45 points in the control condition. Finally, there was a moderate effect favoring MORPH on the emotional well-being subscale that was no longer present in the imputed analysis $\left(F(1,24)=0.07, \eta^{2}\right.$ $=0.00$ ). There were no meaningful effects on the remaining SF-36 subscales. Results from models with imputed data are displayed in Supplemental Table 1, and otherwise do not differ meaningfully from those presented herein.

\section{Discussion}

The MORPH intervention yielded moderate-to-large effects on self-efficacy for walking and satisfaction with physical functioning. MORPH also yielded moderate-to-large effects on HRQOL associated with physical functioning and pain. These results align with previous research employing more intense structured exercise protocols. In a recent study of dietary weight loss delivered alone or with either resistance or aerobic training, older adults who received 6 months of exercise demonstrated 9.7-22.6\% higher self-efficacy for walking and a 1.23-1.69 unit higher satisfaction with function score (both adjusted for baseline levels) relative to those who received dietary weight loss alone [18]. MORPH participants reported $15 \%$ higher self-efficacy for walking and 8.24-unit higher satisfaction with function score compared with control after adjusting for baseline values, sex, and age. MORPH participants also demonstrated an 11.82-unit higher adjusted score on the SF-36 physical function subscale and a 14.03-unit higher score on the bodily pain subscale relative to control. These differences fall above a common minimal clinically important change value of 5 for SF-36 subscales $[27,28]$. MORPH is the first to show these effects while
Table 1 Participant characteristics

\begin{tabular}{llll}
\hline & Intervention $(n=15)$ & Control $(n=13)$ & Overall $(N=28)$ \\
\hline $\begin{array}{l}\text { Age (years; } \mathrm{M} \pm \mathrm{SD}) \\
\text { Sex }(\mathrm{n}, \%)\end{array}$ & $70.12 \pm 5.43$ & $70.32 \pm 5.20$ & $70.21 \pm 5.22$ \\
Female & $13(86.7)$ & & \\
Male & $2(13.3)$ & $9(69.2)$ & $22(78.6)$ \\
Race $(\mathrm{n}, \%)$ & $4(30.8)$ & $6(21.4)$ \\
Black & $3(20)$ & $2(15.4)$ & $5(17.9)$ \\
White & $12(80)$ & $11(84.6)$ & $23(82.1)$ \\
Education $(\mathrm{n}, \%)$ & $2(13.3)$ & & $4(14.3)$ \\
High school graduate & $9(60.0)$ & $2(15.4)$ & $16(57.1)$ \\
College graduate & $4(26.7)$ & $7(53.8)$ & $8(28.6)$ \\
Post-graduate & $98.41 \pm 15.88$ & $4(30.8)$ & $98.64 \pm 16.68$ \\
Weight $(\mathrm{kg})$ & $36.99 \pm 4.84$ & $98.91 \pm 18.22$ & $36.96 \pm 4.54$ \\
BMI $\left(\mathrm{kg} / \mathrm{m}^{2} ; \mathrm{M} \pm \mathrm{SD}\right)$ & & $36.92 \pm 4.36$ & \\
\hline
\end{tabular}

$B M I$ body mass index 
Table 2 Baseline and follow-up values adjusted by sex and age

\begin{tabular}{|c|c|c|c|c|c|c|c|}
\hline & \multicolumn{2}{|l|}{ Baseline } & \multicolumn{2}{|l|}{ Follow-up } & \multirow[b]{2}{*}{$\mathrm{F}^{\mathrm{a}}$} & \multirow[b]{2}{*}{$\mathrm{p}$} & \multirow[b]{2}{*}{$\eta^{2}$} \\
\hline & Intervention & Control & Intervention & Control & & & \\
\hline Self-Efficacy & $45.62(9.50)$ & $44.33(9.12)$ & $59.81(8.67)$ & $43.45(8.33)$ & 2.17 & .16 & .09 \\
\hline Satisfaction with function ${ }^{\mathrm{b}}$ & $11.28(1.93)$ & $14.74(1.85)$ & $20.51(3.02)$ & $15.76(2.90)$ & 4.60 & .04 & .18 \\
\hline \multicolumn{8}{|l|}{ SF 36} \\
\hline Physical functioning & $39.24(6.07)$ & $50.70(5.83)$ & $52.63(6.44)$ & $46.42(6.19)$ & 3.79 & .07 & .15 \\
\hline Physical role limitations & $23.17(8.65)$ & $22.84(8.31)$ & $36.78(11.98)$ & $42.97(11.50)$ & 0.18 & .68 & .01 \\
\hline Emotional role limitations & $72.34(12.57)$ & $71.69(12.07)$ & $71.27(10.11)$ & $72.67(9.71)$ & 0.02 & .89 & .00 \\
\hline Energy/fatigue & $44.39(5.91)$ & $32.48(5.68)$ & $47.95(5.44)$ & $33.05(5.22)$ & 0.15 & .70 & .01 \\
\hline Emotional well-being ${ }^{\mathrm{b}}$ & $76.58(5.19)$ & $78.24(4.99)$ & $75.20(5.16)$ & $76.43(4.96)$ & 1.50 & .23 & .07 \\
\hline Social functioning & $65.42(9.25)$ & $72.30(8.89)$ & $64.31(8.51)$ & $69.48(8.17)$ & 0.02 & .89 & .00 \\
\hline Bodily pain & $39.01(4.56)$ & $43.22(4.38)$ & $54.14(6.34)$ & $43.68(6.09)$ & 4.27 & .05 & .17 \\
\hline Perceptions of general health & $57.24(4.29)$ & $54.09(4.12)$ & $56.29(5.13)$ & $56.51(4.93)$ & 0.48 & .50 & .02 \\
\hline
\end{tabular}

Adjusted mean (SE) is presented for each measure. $p$ and $\eta^{2}$ reflect the group by time interaction

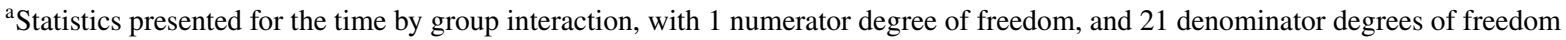

${ }^{\mathrm{b}}$ Original units presented, but a transformation was applied in analyses

focusing on reduced sedentary time and frequent bouts of stepping throughout the day in older adults with chronic pain [9]. One especially novel finding is that our day-long movement prescription resulted in enhanced efficacy for engaging in longer durations of walking. These data are exciting and suggest such an approach to activity promotion may contribute to lasting behavior change, given the role of self-efficacy in behavioral maintenance [7, 29]. Clearly, long-term followup data are needed.

Our results are particularly timely: a large body of evidence underscores the vital role of structured exercise in weight management for older adults [30]. But these findings also echo a general movement in physical activity promotion toward a focus on overall volume of movement and participation in frequent bouts of physical activity throughout the day [31]. Another important contribution of this study is that these benefits were observed from a program that was delivered largely in the home. MORPH integrated a suite of technologies brought together in a custom smartphone app built for older adults that had excellent usability ratings [9] and leveraged a movement protocol that did not require structured exercise supervision or access to exercise equipment. When compared with traditional center-based programming, this approach is likely to be less costly and better able to reach those individuals who may not live near a health center or who have limited transport. The importance of remote interventions with a strong social component was further underscored by the COVID-19 pandemic, which has physically and socially isolated many older adults. The pilot data presented here suggest a technology-facilitated, remote group-mediated intervention that can help to improve several key psychosocial outcomes in older adults with chronic pain.

\section{Limitations and Future Directions}

MORPH was a pilot trial focused on evaluating the acceptability and feasibility of a remotely delivered, group-mediated weight loss intervention tailored for older adults with chronic pain. If well tolerated and feasible, the data provided would facilitate power calculations for a full-scale trial. As such, the main limitation in providing evidence for the efficacy of intervention on pain and social cognitive outcomes under investigation in the current study is the small sample size, and our findings should be interpreted with caution. MORPH also had limited representation of male and non-white participants, and all participants had previously owned a smartphone device. Likewise, only $12 \%$ of screened individuals were eligible for participation, which suggests overly restrictive eligibility criteria or the need for better-tailored recruiting materials.

These pilot results suggest the MORPH intervention may benefit HRQOL, self-efficacy, and satisfaction with function of older adults with chronic multisite pain. A key next step lies in the conduct of a larger trial sufficiently powered to investigate whether changes in self-efficacy mediate the impact of MORPH on activity and weight loss behaviors as well as on HRQOL. Such a study would also benefit from a no-contact maintenance phase to determine whether improvements in self-efficacy and HRQOL persist and predict lasting behavior change. Three of 15 intervention participants did not have complete data, potentially biasing our results. Additionally, we did not collect data on the weight loss intervention alone nor did we collect data on the activity-promotion intervention alone, and so we are unable to determine whether both components meaningfully contribute to the observed effects or the extent to which change in weight and/or physical activity mediated the outcomes of interest in the current 
investigation. Still, we believe these early data show promise for both remote delivery of a group-mediated weight loss and movement intervention for older adults, and the use of a day-long movement prescription for enhancing self-efficacy, satisfaction with functioning, and HRQOL.

\section{Conclusion}

Results of this secondary analysis indicate that a pilot trial leveraging a remote, group-mediated behavioral intervention tailored for older adults with chronic pain contributes to moderate to large improvements in self-efficacy for walking, satisfaction with physical functioning, and both pain and physical functioning components of HRQOL. This approach has the potential for broad scalability and sustainability; additional research is warranted testing the MORPH intervention in a larger and more representative sample collected across a broad geographic area.

Supplementary Information The online version contains supplementary material available at https://doi.org/10.1007/s12529-021-10003-3.

Author contributions Dr. Fanning designed the study, conducted analyses, and prepared the manuscript, Dr. Brooks designed the study and assisted in manuscript preparation, Drs. Rejeski, Nicklas, and Hsieh as well as Mrs. Nesbit and Ford assisted in manuscript preparation and study conduct.

Funding This work was supported by the National Institutes of Health/ National Institute on Aging (Grant Number R21 AG058249-01; AKB and $\mathrm{JF}$ are principal investigators). Dr. Fanning is also supported by the Wake Forest University Claude D. Pepper Older Americans Independence Center (P30-AG21332).

Data Availability Data will be made available upon request to the authors.

\section{Declarations}

Ethics Informed consent was obtained from all individual participants included in the study. All procedures performed in studies involving human participants were in accordance with the ethical standards of the institutional review board and with the 1964 Helsinki declaration and its later amendments or comparable ethical standards. This article does not contain any studies with animals performed by any of the authors. The trial was registered at ClinicalTrials.gov (REDACTED).

Conflict of Interest The authors declare no competing interests.

\section{References}

1. National Center for Health Statistics. Health, United States, 2019: Table 26. Hyattsville, MD. 2021.

2. Rejeski WJ, Marsh AP, Chmelo E, Rejeski JJ. Obesity, intentional weight loss and physical disability in older adults. Obes Rev. 2010;11(9):671-85. PMID:19922431.
3. Katz DA, McHorney CA, Atkinson RL. Impact of obesity on health-related quality of life in patients with chronic illness. $\mathrm{J}$ Gen Intern Med. 2000. PMID:11119171.

4. McAuley E, Doerksen SE, Morris KS, et al. Pathways from physical activity to quality of life in older women. Ann Behav Med. 2008;36(1):13-20. PMID:18677453.

5. Evert AB, Franz MJ. Why weight loss maintenance is difficult. Diabetes Spectr. 2017;30(3):153-6.

6. Deci EL, Ryan RM. Self-determination theory: a macrotheory of human motivation, development, and health. Can Psychol. 2008;49(3):182-5.

7. Bandura A. Self-efficacy: the exercise of control. New York, NY: W. H. Freeman and Company. 1997. ISBN:0716728508.

8. Rejeski W, Fanning J. Models and theories of health behavior and clinical interventions in aging: a contemporary, integrative approach. Clin Interv Aging. 2019;14:1007-19. PMID:31213787.

9. Fanning J, Brooks A, Ip E, Nicklas B, Rejeski W, Nesbit B, Ford S. A mobile health behavior intervention to reduce pain and improve health in older adults with obesity and chronic pain: the MORPH pilot trial. Front Digit Heal. 2020;2(598456).

10. Marlatt G. Determinants of relapse: Implications for the maintenance of behavior change. In: Davidson, PO and Davidson SM, editors. Behavioral Medicine: Changing Health Lifestyles. New York, NY: Brunner-Mazel. 1989.

11. Brantley P, Appel L, Hollis J, et al. Design considerations and rationale of a multi-center trial to sustain weight loss: the weight loss maintenance trial. Clin Trials. 2008;5(5):546-56.

12. Fanning J, Brooks AK, Ip E, Nicklas BJ, Rejeski WJ. A Mobile Health Intervention to Reduce Pain and Improve Health (MORPH) in older adults with obesity: protocol for the MORPH Trial. JMIR Res Protoc. 2018;7(5):e128.

13. Rejeski WJ, Ambrosius WT, Burdette JH, Walkup MP, Marsh AP. Community weight loss to combat obesity and disability in at-risk older adults. J Gerontol A Biol Sci Med Sci. 2017;glw252.

14. Bowen S, Chawla N, Collins SE, et al. Mindfulness-based relapse prevention for substance use disorders: a pilot efficacy trial. Subst Abus. 2009;30(4):295-305.

15. Ware J, Kosinski M, Keller SD. A 12-item short-form health survey: construction of scales and preliminary tests of reliability and validity. Med Care. 1996;34:220-33.

16. Paxton RJ, Motl RW, Aylward A, Nigg CR. Physical activity and quality of life-the complementary influence of self-efficacy for physical activity and mental health difficulties. Int $\mathrm{j}$ Behav Med. 2010;17(4):255-63.

17. Fontaine KR, Barofsky I. Obesity and health-related quality of life. Obes Rev. 2001;2(3):173-182.

18. Fanning J, Walkup MP, Ambrosius WT, et al. Change in healthrelated quality of life and social cognitive outcomes in obese, older adults in a randomized controlled weight loss trial: does physical activity behavior matter? J Behav Med. 2017;41(3):1-10.

19. Conner M, Norman P. Health behaviour: current issues and challenges. Psychol Health. 2017;32(8):895-906.

20. McAuley E, Blissmer B, Katula J, Duncan TE. Exercise environment, self-efficacy, and affective responses to acute exercise in older adults. Psychol Health. 2000;15(3):341-55.

21. Ray KM, Hector LL, Lynes LK, Stewart AL, Painter PL, Dibble SL. Assessment of satisfaction with physical fitness in kidney transplant recipients. Med Sci Sport Exerc. 1996;28(5):7.

22. RAND Corporation. 36-Item Short Form Survey (SF-36) Scoring instructions. 2016. PMID:23770012

23. LoMartire R, Äng BO, Gerdle B, Vixner L. Psychometric properties of short form-36 health survey, EuroQol 5-dimensions, and hospital anxiety and depression scale in patients with chronic pain. Pain. 2020;161(1):83-95.

24. Dohrn IM, Gardiner PA, Winkler E, Welmer AK. Device-measured sedentary behavior and physical activity in older adults differ by 
demographic and health-related factors. Eur Rev Aging Phys Act. 2020;17(1)

25. Cohen J. Statistical power analysis for the behavioral sciences. New York, NY: Academic Press. 1988.

26. Rubin LH, Witkiewitz K, Andre JS, Reilly S. Methods for handling missing data in the behavioral neurosciences: don't throw the baby rat out with the bath water. J Undergrad Neurosci Educ. 2007;5(2):71-7.

27. Busija L, Osborne RH, Nilsdotter A, Buchbinder R, Roos EM. Magnitude and meaningfulness of change in SF-36 scores in four types of orthopedic surgery. Health Qual Life Outcomes. 2008;6:55.

28. Wyrwich KW, Tierney WM, Babu AN, Kroenke K, Wolinsky FD. A comparison of clinically important differences in health-related quality of life for patients with chronic lung disease, asthma, or heart disease. Health Serv Res. 2005;40(2):577-91.
29. Higgins TJ, Middleton KR, Winner L, Janelle CM. Physical activity interventions differentially affect exercise task and barrier selfefficacy: a meta-analysis. Health Psychol. 2014;33(8):891-903.

30. Villareal DT, Chode S, Parimi N, et al. Weight loss, exercise, or both and physical function in obese older adults. N Engl J Med. 2011;364(13):1218-29.

31. Piercy KL, Troiano RP, Ballard RM, Carlson SA, Fulton JE, Galuska DA, George SM, Olson RD. The physical activity guidelines for Americans. JAMA. 2018;320(19):2020-8.

Publisher's Note Springer Nature remains neutral with regard to jurisdictional claims in published maps and institutional affiliations. 